\title{
Isolation of Bacteroides corrodens from infections in children
}

\author{
H. B. MARSDEN AND W. A. HYDE \\ Royal Manchester Children's Hospital, Pendlebury, Lancs
}

SYNOPSIS Six cases of infections in children with Bacteroides corrodens are reported. In five the infection was mixed but in one fatal extradural and subdural empyema the organism was found in pure culture. The bacteriological findings are presented. It is suggested that Bacteroides corrodens may assume a pathogenic role and the rarity of its isolation may be due to delay in the appearance of the colony.

Bacteroides corrodens was first described by Henriksen (1948) who isolated Gram-negative anaerobic organisms with 'pitted' colonies from a pulmonary and a perianal abscess. Holm (1950) gave the name of 'corroding bacillus' to this organism because of the nature of the growth. Eiken (1958) made a more comprehensive study of a number of strains, mainly from infections of the oral region, including submental, buccal, mandibular, and parotid abscesses, with one strain from a cerebral abscess. In practically all of the cases other anaerobic and sometimes aerobic bacteria were present. Similarly, mixed infections were present in Henriksen's cases (1948), and Holm (1950) in an extensive study on the aetiology of human actinomycosis found the "corroding bacillus' to be the most frequent concomitant organism.

More recently Khairat (1967) isolated Bacteroides corrodens from blood cultures taken one minute after dental extraction and Henriksen (1969) found a further 26 strains in nose and throat swabs. Henriksen considered that the organism did not fit into the genus Bacteroides and that correct classification should await further study. Hill, Snell, and Lapage (1970), in a study of the identification and characterization of Bacteroides corrodens which includes two of the strains from the present series, believe that it is premature to make any decision about the taxonomy of this organism.

At the present time there are no reports of the isolation of Bacteroides corrodens from patients in the British Isles. In addition, the previous reports have suggested that this organism is comparatively harmless but its isolation in pure culture from a fatal Received for publication 30 May 1970. subdural empyema encountered in our series would indicate that more attention should be given to its pathogenicity.

\section{Bacteriology}

The bacteriological characteristics of Bacteroides corrodens are described by Khairat (1967) and Hill et al (1970).

The striking feature in our material, as in the other reported series, is the colonial appearance. There are two types of colony which are often found together on primary isolation and only appear after $\mathbf{4 8}$ hours' incubation. After about three days on blood agar, they are about $1.5 \mathrm{~mm}$ in diameter. One has lacunar formation with a raised centre and is not unlike a larger version of the colony of Mycoplasma spp. The other is glistening and umbonate with a lobate margin. All of our strains were facultative anaerobes showing improved growth in $\mathrm{CO}_{2}$ although strictly anaerobic strains have been reported (Henriksen, 1948; Khairat, 1967). Factors $X$ and V were not required but the growth was enhanced by the addition of factor X. Hugh and Leifson's medium showed little growth and no reaction; catalase was negative. Using Kovac's method the organisms have all been oxidase negative in our experience, although the same strains have been considered to be oxidase positive by other workers using the same technique and materials (Hill, personal communication). Khairat (1967) regarded his 19 strains as oxidase negative and it would seem that too much reliance should not be placed on this test for routine diagnosis. Differentiation from Brucella spp may be made by the negative urease activity of Bacteroides corrodens, 
and lysine decarboxylase is recommended for distinguishing Brucella spp and Moraxella spp (Hill et al, 1970).

The antibiotic sensitivity pattern of our strains has been as follows: all except one were sensitive to penicillin, two were resistant to erythromycin, and all were resistant to novobiocin, and sensitive to tetracycline, chloramphenicol, ampicillin, gentamicin, streptomycin, trimethoprim/sulphonamide, colistin methane sulphonate, and carbecillinin.

\section{Case Reports}

CASE 1

A boy aged 9 years was admitted on 25 December 1966.

The child had been unwell for three weeks with vomiting and had been drowsy and irritable for two days before admission. No antibiotics had been given during this time. On examination he was feverish, drowsy, and irritable with neck stiffness and photophobia. Radiographs showed opacity of the right maxillary and frontal sinuses. Jacksonian fits developed and electroencephalography and angiography indicated a right-sided extradural frontal abscess. Treatment by drainage with instillation of penicillin and streptomycin produced temporary improvement but the fits recurred and reexploration showed a subdural empyema on the same side. In spite of the second exploration and evacuation the patient died on the following day. At necropsy there was cerebral swelling with cerebellar coning and herniation of the brain through the craniotomy with infarction of the cerebral cortex. A subdural empyema with greenish pus extended over the whole of the right hemisphere.

Bacteroides corrodens was isolated from the extradural abscess at the first operation and from the subdural empyema at necropsy. Blood culture at the time of the first operation was sterile.

CASE 2

A girl aged 14 years was admitted on 14 May 1968. The patient had had nasal catarrh for two weeks following an attack of 'flu'. There had been vomiting, fits, and frontal headache, and she was toxic and drowsy. Apnoeic attacks had been noted and on admission the pupils were dilated and fixed. Angiography failed to show any intracranial circulation. Craniotomy was performed and $10 \mathrm{ml}$ of foul green pus was aspirated from an abscess at a depth of $7 \mathrm{~cm}$ in the left frontal region. Penicillin, 500,000 units, was instilled but spontaneous respiration did not develop and the child died on the following day.

At necropsy there was bilateral frontal sinusitis with caries of the frontal bone and bilateral brain abscesses in the frontal region.

A Gram film from the pus obtained at operation showed masses of Gram-negative bacilli with a few $\overline{0}$ chains of streptococci, and culture yielded a heavy $\underline{ }$ growth of Bacteroides corrodens of both colonial types and an anaerobic streptococcus.

\section{CASE 3}

A little girl aged 2 years was admitted on 18 January 1969.

The child had a colostomy as a result of an abscess $\stackrel{\omega}{\circ}$ and perforation in the region of the splenic flexure $\overline{ }$ in early infancy. The colostomy was closed and the is bowel refashioned at 2 years of age. Repeated $+\underset{\sim}{+}$ attacks of partial obstruction led to re-exploration several weeks later. At this time there was necrosis $\vec{V}$ and inflammation at the site of anastomosis. The $O$ colostomy was reformed but a purulent discharge $\underset{\neg}{\longrightarrow}$ from the wound occurred which cleared up without 3 antibiotic treatment.

A swab from the wound yielded a heavy growth of $\frac{\widehat{\rho}}{\partial}$ both colonial types of Bacteroides corrodens in $\vec{\varphi}$ addition to Escherichia coli.

\section{CASE 4}

A boy aged 13 months was admitted on 15 May 1969 .

The child had had a purulent nasal discharge with $\overline{0}$

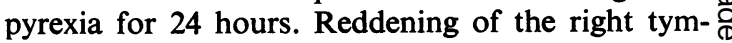
panic membrane was noted and there was a slight 0 cough as well as malaise.

Argotone drops were instilled into the nose and oral penicillin was given. The temperature settled and the patient was discharged after 10 days.

A profuse growth of both types of colony of Bacteroides corrodens was found in pure culture from a nasal swab taken shortly after admission.

\section{CASE 5}

A boy aged 16 months was admitted on 19 Mayo 1969 with anorexia, malaise, and pyrexia. The right leg was flexed and there was guarding over the righto side of the abdomen. A mass was palpated in the right loin and there was leucocytosis. The urine dido not show any abnormality. A right perinephric N abscess was drained and $100 \mathrm{ml}$ of pus evacuated. N Penicillin and streptomycin were given intra- $\omega$ muscularly and a drain was inserted. Convalescence was uneventful.

A heavy growth of Bacteroides corrodens was grown from the pus as well as Escherichia coli and ${ }_{T}^{+}$ Streptococcus faecalis.

CASE 6

A boy aged 7 years was admitted on 4 June 1969 . The patient had collided with another boy while 
playing cricket and sustained a laceration over the right side of the forehead. A tooth was removed from the wound and the latter sutured. Tetanus toxoid was given. The following day the patient was unwell with a discharge of pus from the wound and swelling and oedema of the eyelids. Intramuscular penicillin and streptomycin were given and the discharge cleared up after four days.

A Gram film of the pus from the wound showed polymorphonuclear leucocytes and large numbers of Gram-negative bacilli, with a few Gram-positive cocci and bacilli. The culture yielded a profuse growth of normal upper respiratory tract flora with Bacteroides corrodens.

\section{Discussion}

The reports of isolations of Bacteroides corrodens suggest that this organism is widely distributed in human mucous membranes. Identification is easy and the paucity of published reports is probably due to the delay in the appearance of the colonies. The pathogenicity of the organism has been doubted (Henriksen, 1969) and Bacteroides corrodens has usually been associated with other bacteria when isolated from human infections. However, in case 1 of the present series small Gram-negative bacilli only were seen in the operation specimen and a profuse pure culture of Bacteroides corrodens was isolated from the craniotomy and necropsy material. Most of the infections with which Bacteroides corrodens has been associated have involved the oropharyngeal region and respiratory tract but it is probable that the organism may occur in any human infection which is related to a mucous membrane, including infections in the region of the gastrointestinal tract.

We should like to express our thanks to Dr S. P. Lapage and Mr L. R. Hill of the National Collection of Type Cultures for the initial identification of the organism and for their advice.

\section{References}

Eiken, M. (1958). Studies on an anaerobic rod-shaped Gramnegative microorganism: Bacteroides corrodens N.S.P. Acto path. microbiol. scand., 43, 404-416.

Henriksen, S. D. (1948). Studies in Gram-negative anaerobes. II. Gram-negative anaerobic rods with spreading colonies. Acta path. microbiol. scand., 25, 368-375.

Henriksen, S. D. (1969). Corroding bacteria from the respiratory tract. Acta path. microbiol. scand., 75, 91-96.

Hill, L. R., Snell, J. J. S., and Lapage, S. P. (1970). Identification and characterisation of Bacteroides corrodens. J. med. Microbiol., 3, 483-491.

Holm, P. (1950). Studies on the aetiology of human actinomycosis. I. The 'other microbes' of actinomycosis and their importance. Acta path. microbiol. scand., 27, 736-751.

Khairat, O. (1967). Bacteroides corrodens isolated from bacteriaemias. J. Path. Bact., 95, 29-40. 